\title{
Sobre as funções racionais multi-sequenciais
}

\author{
Rodrigo de Souza ${ }^{1 *}$ \\ ${ }^{1}$ Departamento de Computação - Universidade Federal Rural de Pernambuco (UFRPE) \\ rodrigo.npmsouza@ufrpe.br
}

\begin{abstract}
Multi-sequential functions were introduced by Choffrut and Schützenberger as the family of rational functions whose graph is a finite union of sequential functions. Recently, Jeckert and Filiot have shown that it is decidable in polynomial time if a rational function is multi-sequential. In this communication we study the rational functions that are a union of $k$ sequential functions, for a fixed integer $k$. We present three characterizations of these functions, which are related to classical results of the theory of rational relations.
\end{abstract}

Resumo. Funções multi-sequenciais foram introduzidas por Choffrut $e$ Schützenberger como a família das funções racionais cujo gráfico é uma união finita de funções sequenciais. Recentemente, Jeckert e Filiot mostraram que é decidivel em tempo polinomial se uma função racional é multi-sequencial. Nesta comunicação estudamos as funções que são uma união de $k$ funções sequenciais, para um inteiro fixo $k$. Apresentamos três caracterizações dessas funções, que recordam resultados clássicos sobre as relações racionais.

\section{Introdução}

A elucidação da estrutura de famílias de objetos realizados por autômatos - linguagens, séries, relações - decompondo-os em objetos mais elementares é uma das linhas de estudo mais tradicionais na área da Teoria dos Autômatos. Por exemplo, no tratado clássico de Samuel Eilenberg [Eilenberg 1974], um capítulo é dedicado à estrutura dos conjuntos reconhecíveis, e um algoritmo de decomposição dessas linguagens é apresentado.

Nossa contribuição filia-se a essa linha ao considerar a estrutura das funções entre palavras realizadas por autômatos com entrada e saída - os transdutores. Um transdutor ${ }^{1}$ é uma extensão do modelo de autômato, no qual as transições são rotuladas por um par de palavras, uma dita de entrada e outra de saída. Ao invés de reconhecer linguagens, os transdutores realizam relações entre monóides livres $A^{*}$ e $B^{*}$; essas relações são precisamente as relações racionais de $A^{*}$ a $B^{*}$ (= subconjuntos do produto cartesiano $A^{*} \times B^{*}$ que podem ser obtidos com aplicações das operações de união, produto e estrela a partir de unitários). Uma função racional é uma relação racional que é uma função parcial. ${ }^{2}$

Em diversos aspectos, relações racionais são objetos muito mais intrincados do que as linguagens reconhecíveis. ${ }^{3}$ Por outro lado, as funções racionais, que estudamos aqui, exibem propriedades notáveis e por isso ganharam muita atenção na literatura.

\footnotetext{
*Este trabalho é apoiado pelo projeto Problemas estruturais em modelos formais de Computação, Edital MCTI/CNPQ/Universal 14/2014 (Processo 459957/2014-7).

${ }^{1}$ Por limitação de espaço, não apresentamos neste resumo definições e notações básicas sobre autômatos e transdutores. Esse material pode ser consultado no livro de Jacques Sakarovitch [Sakarovitch 2009].

${ }^{2}$ Ao longo do texto, $A$ e $B$ aparecerão livremente e representam dois alfabetos.

${ }^{3}$ Por exemplo, a equivalência não é decidível para relações racionais.
} 
O problema abordado nesta comunicação refere-se ao conceito de determinismo em transdutores. Contrariamente aos autômatos sobre um monóide livre, nem todo transdutor funcional (= realiza uma função) pode ser determinizado. A caracterização das funções racionais que podem ser realizadas por um transdutor sequencial, ou seja, determinístico na entrada - são as chamadas funções sequenciais - é um resultado profundo, obtido por Choffrut em 1979 [Choffrut 1979, Lombardy and Sakarovitch 2006].

Em 1986, Choffrut e Schützenberger consideraram um relaxamento da sequencialidade: mesmo que uma função racional não seja sequencial, pode-se escrevê-la como uma união finita de funções sequenciais? Chamamos de multi-sequencial uma tal função. ${ }^{4}$ O principal resultado apresentado em [Choffrut and Schützenberger 1986] é uma caracterização estrutural dos transdutores que realizam uma função multi-sequencial. Os problemas de decidir se uma função racional é multi-sequencial e de decompor uma tal função em uma união de funções sequenciais foram considerados por Jecker e Filiot [Jecker and Filiot 2015], com um algoritmo polinomial para o primeiro problema.

Nesta comunicação, consideramos uma versão parametrizada, por um inteiro $k>0$, da questão introduzida por Choffrut e Schützenberger: que funções racionais podem ser escritas como uma união de $k$ funções sequenciais? Chamamos tais funções de $k$-sequenciais. A semelhança com os resultados mencionados é apenas aparente: os trabalhos de Choffrut, Schützenberger, Jeckert e Filiot tratam de investigar a existência de uma decomposição; aqui, deseja-se uma resposta mais refinada, ao fixar-se um tamanho para a decomposição. Essa diferença pode surpreendentemente ser posta em paralelo com dois problemas de decisão clássicos para transdutores: decidir se um transdutor é $k$-valorado, para um dado inteiro $k$ (ou seja, se a imagem de toda palavra no domínio é um conjunto de no máximo $k$ palavras); decidir se é finitamente valorado (se existe um tal $k$ ). Ambos os problemas podem ser decididos em complexidade polinomial, o que demonstramos com J. Sakarovitch através de construções estruturais com transdutores [Sakarovitch and de Souza 2008]. Mas as construções empregadas são muito diferentes.

Nossa contribuição consiste de três caracterizações das funções $k$-sequenciais, que referem-se a facetas distintas desses objetos. Cada uma relaciona-se com um resultado clássico sobre transdutores: a primeira, Teorema 3.1, recorda a supracitada caracterização, de sabor topológico, de Choffrut das funções sequenciais (Teorema 2.1); a segunda, Teorema 3.2, também faz referência ao trabalho de Choffrut (Teorema 2.2), mas é mais estrutural, ao lidar com propriedades de passeios em transdutores; finalmente, a terceira, Teorema 3.3, evoca uma propriedade de passeios de transdutores $k$-valorados implícita em [Weber 1996] e retrabalhada em [Sakarovitch and de Souza 2010] (Teorema 2.3). Por restrições de espaço, limitamo-nos a expor, com um mínimo de notação, os enunciados dos três resultados clássicos referidos, e, em seguida, enunciar nossos resultados. Concluímos com um comentário sobre consequências dos mesmos a serem investigadas.

\section{Funções sequenciais e relações $k$-valoradas: propriedades clássicas}

A principal notação envolvida nos resultados que vamos expor é uma métrica sobre um monóide livre; essa função associa a todo par de palavras $u, v \in A^{*}$ a soma dos comprimentos dos sufixos respectivos após retirar-se o maior prefixo comum entre $u$ e $v$.

\footnotetext{
${ }^{4} \mathrm{~A}$ função que associa toda palavra da forma $u_{1} x_{1} \# u_{2} x_{2} \# \ldots u_{k} x_{k} \#$ a $x_{1} u_{1} \# x_{2} u_{2} \# \ldots x_{k} u_{k} \#$, onde, para todo $i, u_{i} \in\{a, b\}^{*}$ e $x_{i} \in\{a, b\}$, é um exemplo de função racional que não é multi-sequencial.
} 
Podemos definir essa métrica formalmente com o grupo livre sobre $A$ : $\|u, v\|=\left|u^{-1} v\right|$.

Definição 2.1 (Função lipschitziana) Uma função $f: A^{*} \rightarrow B^{*}$ é lipschitziana se existe um inteiro $L \geq 0$ tal que, para todo u e v no domínio de $f$, $\| u f$, vf $\|\leq L\| u, v \|$.

Não é difícil demonstrar que toda função sequencial é lipschitziana. A substância da caracterização de Choffrut é a recíproca, cuja demonstração é intrincada:

Teorema 2.1 (Choffrut 1978) Uma função racional é sequencial sse é lipschitziana.

Uma caracterização mais estrutural e efetiva das funções sequenciais, também presente no trabalho de Choffrut, pode ser expressa através de propriedades do produto cartesiano de um transdutor $\mathcal{T}$ por ele mesmo: em $\mathcal{T}^{2}$, um passeio representa simultaneamente dois passeios de $\mathcal{T}$ com a mesma entrada, em $\mathcal{T}^{3}$, três passeios, etc. Veja detalhes desse produto em [Sakarovitch and de Souza 2010]. Precisamos da seguinte definição:

Definição 2.2 (Passeio $(i, j)$-conjugado) Seja c um passeio de $\mathcal{T}^{k+1}$ de um estado inicial $\left(p_{1}, p_{2}, \ldots, p_{k+1}\right)$ ao estado $\left(q_{1}, q_{2}, \ldots, q_{k+1}\right)$; sejam $p_{i} \stackrel{u \mid u_{1}}{\longrightarrow} q_{i}$ e $p_{j} \stackrel{u \mid u_{2}}{\longrightarrow} q_{j}$ as projeções de c nos índices $i$ e $j$, respectivamente. Dizemos que cé $(i, j)$-conjugado se, para todo circuito d que começa e termina em $\left(q_{1}, q_{2}, \ldots, q_{k+1}\right)$, as projeções de d nos índices i e $j$, $q_{i} \stackrel{v \mid v_{1}}{\longrightarrow} q_{i}$ e $q_{j} \stackrel{v \mid v_{2}}{\longrightarrow} q_{j}$, satisfazem $u_{1}^{-1} u_{2}=v_{1}^{-1} u_{1}^{-1} u_{2} v_{2}$.

A caracterização estrutural de Choffrut é a base de algoritmos para decidir se uma função racional é sequencial (detalhes podem ser vistos em [Béal et al. 2003]):

Teorema 2.2 (Choffrut 1978) Um transdutor funcional $\mathcal{T}$ realiza uma função sequencial sse todo passeio de $\mathcal{T}^{2}$ que começa em um estado inicial é (1,2)-conjugado.

Relativamente às relações racionais $k$-valoradas, a propriedade que nos interessa envolve o conceito de deslocamento entre passeios $c$ e $d$ com a mesma entrada e que começam em um estado inicial: considerando-se todos os prefixos de $c$ e $d$ de mesmo comprimento, o deslocamento é o máximo entre as diferenças das saídas desses prefixos.

Definição 2.3 (Deslocamento) Em um transdutor $\mathcal{T}$, o deslocamento entre passeios $c$ e d com a mesma entrada, e que começam em um estado inicial, é o inteiro $\langle c, d\rangle=$ $\max \{\|x, y\| \mid p \stackrel{u \mid x}{\longrightarrow}$ q prefixo de $c, r \stackrel{u \mid y}{\longrightarrow}$ s prefixo de $d\}$.

Se um transdutor $\mathcal{T}$ é $k$-valorado, não somente toda tupla de $k+1$ passeios bemsucedidos com a mesma entrada tem pelo menos um par com a mesma saída (isso é uma paráfrase da definição), mas necessariamente existe um par cujo deslocamento é limitado:

Teorema 2.3 (Weber 1996) Um transdutor $\mathcal{T}$ é $k$-valorado se, e somente se, existe um inteiro $L$ tal que, para todo passeio bem-sucedido $c$ de $\mathcal{T}^{k+1}$, existem duas coordenadas i e $j$ tais que as projeções $c_{i}$ et $c_{j}$ tem a mesma saída e satisfazem $\left\langle c_{i}, c_{j}\right\rangle<L$.

\section{Contribuições}

Teorema 3.1 Uma função racional $f: A^{*} \rightarrow B^{*}$ é $k$-sequencial se, e somente se, existe um inteiro $L$ tal que todo conjunto $u_{1}, u_{2}, \ldots, u_{k+1}$ de palavras no domínio de $f$ admite indices $i$ e $j$ tais que $\left\|u_{i} f, u_{j} f\right\| \leq L\left\|u_{i}, u_{j}\right\|$. 
Teorema 3.2 Um transdutor funcional $\mathcal{T}$ realiza uma função $k$-sequencial se, e somente se, todo passeio c de $\mathcal{T}^{k+1}$, que começa em um estado inicial, satisfaz: para todo prefixo $c^{\prime}$ de $c$, existe pelo menos um par $i, j$ de indices tais que $c^{\prime} e ́(i, j)$-conjugado.

Teorema 3.3 Um transdutor funcional $\mathcal{T}$ realiza uma função $k$-sequencial se, e somente se, existe um inteiro $L$ tal que, para todo passeio $c$ de $\mathcal{T}^{k+1}$ que começa em um estado inicial, existem coordenadas $i$ e $j$ tais que as projeções $c_{i}$ e $c_{j}$ satisfazem $\left\langle c_{i}, c_{j}\right\rangle<L$.

\section{Comentários}

As demonstrações dos teoremas 3.1, 3.2 e 3.3 são relacionadas. Por exemplo, no Teorema 3.2, a existência de um passeio que não satisfaz as condições enunciadas permite a construção de um conjunto de palavras que não satisfaz as condições do Teorema 3.1; a existência de um passeio que não satisfaz as condições do Teorema 3.3 permite construir um passeio que não satisfaz as condições do Teorema 3.2.

A sequência natural de nossos resultados é a investigação de um algoritmo para decidir se uma função é $k$-sequencial com base no Teorema 3.2 e de uma decomposição de uma função $k$-sequencial em $k$ transdutores sequenciais partindo do Teorema 3.3.

Por fim, agradecemos aos revisores anônimos pela leitura cuidadosa da versão preliminar deste texto, que nos permitiu melhorá-lo substancialmente.

\section{Referências}

Béal, M. P., Carton, O., Prieur, C., and Sakarovitch, J. (2003). Squaring transducers: an efficient procedure for deciding functionality and sequentiality. Theoretical Computer Science, 292(1):45-63.

Choffrut, C. (1979). A generalization of Ginsburg and Rose's characterization of gsm mappings. In ICALP'79, volume 71 of Lecture Notes in Computer Science, pages 88-103. Springer-Verlag.

Choffrut, C. and Schützenberger, M. P. (1986). Décomposition de fonctions rationnelles. In STACS 86, volume 210 of Lecture Notes in Computer Science, pages 213-226. Springer.

Eilenberg, S. (1974). Automata, Languages, and Machines. Academic Press, Inc.

Jecker, I. and Filiot, E. (2015). Multi-sequential word relations. In DLT 2015, volume 9168 of Lecture Notes in Computer Science, pages 288-299. Springer.

Lombardy, S. and Sakarovitch, J. (2006). Sequential? Theoretical Computer Science, 356(1-2):224-244.

Sakarovitch, J. (2009). Elements of Automata Theory. Cambridge University Press.

Sakarovitch, J. and de Souza, R. (2008). On the decidability of bounded valuedness for transducers. In MFCS 2008, volume 5162 of Lecture Notes in Computer Science, pages 588-600. Springer.

Sakarovitch, J. and de Souza, R. (2010). Lexicographic decomposition of k-valued transducers. Theory of Computing Systems, 47(3):758-785.

Weber, A. (1996). Decomposing A $k$-valued transducer into $k$ unambiguous ones. ITA, 30(5):379-413. 